\title{
Crystal Structure of 2,6-Dicarbomethoxy-5-pivaloyloxymethyl-1,3- benzenediol
}

\author{
Adrián Covarrubias-Zúñiga ${ }^{\dagger}$, Simón Hernández-OrTega, Armando GonZález-LuCaS \\ and Noé ZúÑIGA-VILLARREAL
}

Instituto de Química, Universidad Nacional Autónoma de México,

Circuito Exterior, Ciudad Universitaria, Coyoacan 04510, México, D. F. México

Although the synthetic approaches to highly substituted phenols are limited ${ }^{1-6}$, the construction of these aromatic systems is of great synthetical utility. We developed a route to highly substituted aromatic rings starting from aliphatic compounds; $;^{7}$ using our method, we could obtain 2,6-dicarbomethoxy-5-pivaloyloxymethyl-1,3-benzenediol, 1 (Fig. 1). The molecular structure was confirmed by X-ray diffraction. Data were collected on a Siemens P4 diffractometer. Crystallographic data, details of an X-ray data collection and a structure refinement as well are summarized in Table 1. The intensities were collected using nickelfiltered $\mathrm{Cu} \mathrm{K}_{\alpha}$ radiation $(\lambda=1.54178 \AA$ ) at room temperature, and were corrected for Lorentz and polarization effects. ${ }^{8}$ The structure was solved by direct methods and refined by full-matrix least-squares with anisotropic temperature factors for the non-hydrogen atoms. ${ }^{9}$ The hydrogen atoms were placed at their idealized positions and included in a structure-factor calculation with fixed isotropic thermal parameters $\left(U=0.06 \AA^{2}\right)$. The hydroxyl $\mathrm{H}$-atoms were located on a difference Fourier map, and their positional parameters were refined.

A diagram of the structure of compound $\mathbf{1}$ is shown in Fig. 2. The bond lengths and angles (Tables 2 and 3) agree with the expected values. Two intramolecular hydrogen bonds $[\mathrm{H} 4 \cdots \mathrm{O} 5,1.67(5) \AA \AA$; O $4 \cdots \mathrm{O} 5,2.490(4) \AA$;

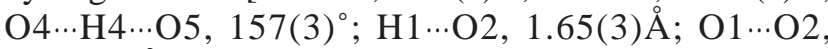
$2.551(4) \AA$; $\left.\mathrm{O} 1 \cdots \mathrm{H} 1 \cdots \mathrm{O} 2,148(2)^{\circ}\right]$ keep the carbonyl

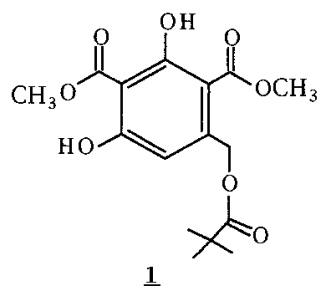

Fig. 1 Structure of 2,6-dicarbomethoxy-5-pivaloyloxymethyl1,3-benzenediol.

\footnotetext{
$\doteqdot$ To whom correspondence should be addressed.
}

Table 1 Crystallographic data for 2,6-dicarbomethoxy-5pivaloyloxymethyl-1,3-benzenediol

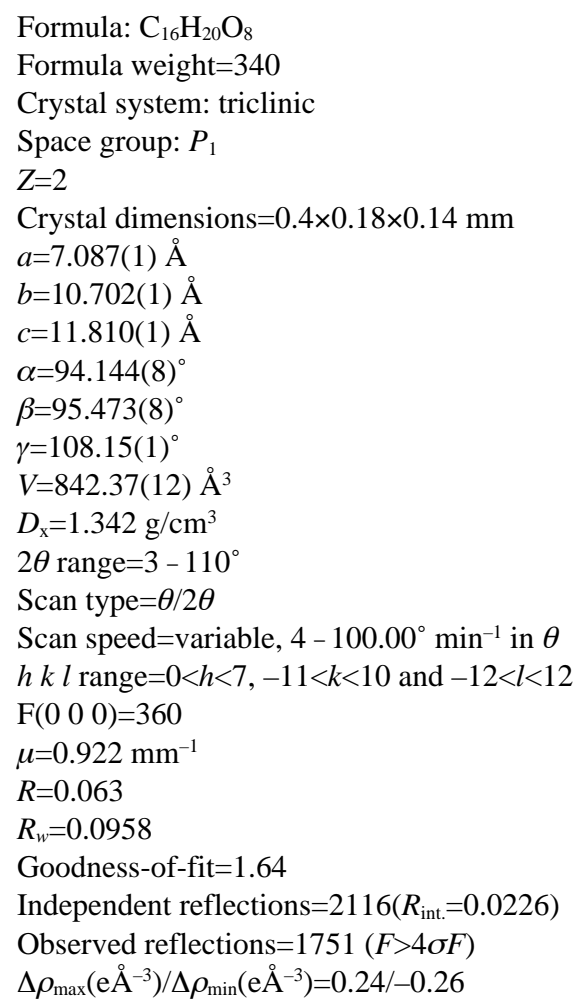

and hydroxy groups coplanar, and at the same time account for the low frequency observed for the stretching carbonyl absorption in the IR spectrum $\left(1660 \mathrm{~cm}^{-1}\right)$.

Contribution No. 1629 of the Instituto de Quúmica, UNAM. This research was supported by a Grant-in-Aid for Scientific Research (No. 40361-5-3352E) from CONACYT, México.

\section{References}

1. Y. Ozaki and S.-W. Kim, Chem. Pharm. Bull., 37, 304 (1989); Y. Ozaki, I. Oshio and S.-W. Kim, Chem. Pharm. Bull., 37, 1434 (1989); Y. Ozaki, I. Oshio, Y. Ohsuga, S. 


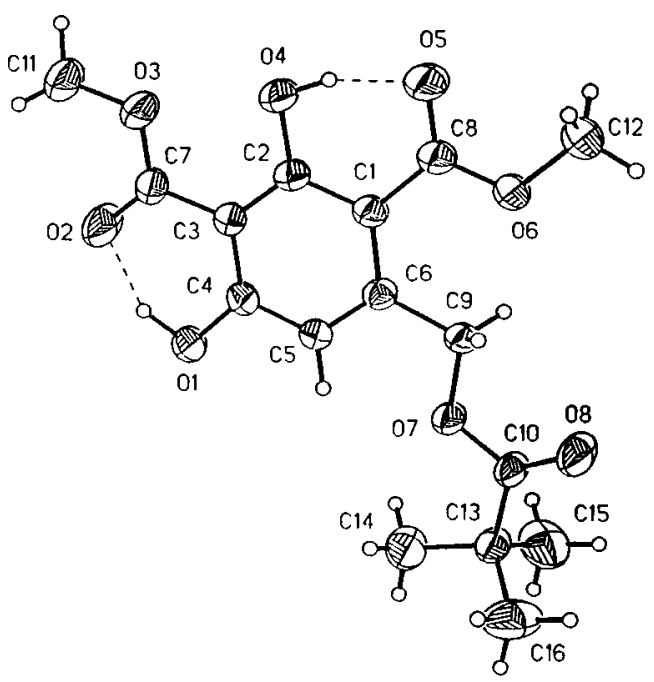

Fig. 2 Molecular structure of compound 1, showing the numbering scheme for the atoms.

Table 2 Atomic coordinates $\left(\times 10^{4}\right)$ and equivalent isotropic displacement coefficients $\left(\AA^{2} \times 10^{3}\right)$

\begin{tabular}{lllll}
\hline & $x$ & \multicolumn{1}{c}{$y$} & \multicolumn{1}{c}{$z$} & $U(\mathrm{eq})$ \\
\hline $\mathrm{O}(1)$ & $779(5)$ & $7186(2)$ & $10969(2)$ & $70(1)$ \\
$\mathrm{O}(2)$ & $264(8)$ & $5759(3)$ & $9068(3)$ & $110(2)$ \\
$\mathrm{O}(3)$ & $1043(5)$ & $6595(3)$ & $7487(2)$ & $69(1)$ \\
$\mathrm{O}(4)$ & $2320(5)$ & $9095(3)$ & $7519(2)$ & $71(1)$ \\
$\mathrm{O}(5)$ & $3605(6)$ & $11550(3)$ & $7594(2)$ & $89(2)$ \\
$\mathrm{O}(6)$ & $4237(4)$ & $12815(2)$ & $9238(2)$ & $61(1)$ \\
$\mathrm{O}(7)$ & $3189(4)$ & $11612(2)$ & $12374(2)$ & $52(1)$ \\
$\mathrm{O}(8)$ & $4156(7)$ & $13741(3)$ & $12968(2)$ & $95(2)$ \\
$\mathrm{C}(1)$ & $2894(5)$ & $10505(3)$ & $9280(3)$ & $41(1)$ \\
$\mathrm{C}(2)$ & $2248(5)$ & $9234(3)$ & $8644(3)$ & $45(1)$ \\
$\mathrm{C}(3)$ & $1558(5)$ & $8074(3)$ & $9176(3)$ & $45(1)$ \\
$\mathrm{C}(4)$ & $1467(5)$ & $8210(3)$ & $10360(3)$ & $47(1)$ \\
$\mathrm{C}(5)$ & $2084(5)$ & $9453(3)$ & $10983(3)$ & $45(1)$ \\
$\mathrm{C}(6)$ & $2776(4)$ & $10586(3)$ & $10471(3)$ & $40(1)$ \\
$\mathrm{C}(7)$ & $904(6)$ & $6714(3)$ & $8583(3)$ & $54(1)$ \\
$\mathrm{C}(8)$ & $3590(6)$ & $11647(3)$ & $8632(3)$ & $50(1)$ \\
$\mathrm{C}(9)$ & $3445(5)$ & $11905(3)$ & $11217(3)$ & $42(1)$ \\
$\mathrm{C}(10)$ & $3656(6)$ & $12629(3)$ & $13180(3)$ & $52(1)$ \\
$\mathrm{C}(11)$ & $397(8)$ & $5270(4)$ & $6916(4)$ & $78(2)$ \\
$\mathrm{C}(12)$ & $4920(9)$ & $13957(4)$ & $8610(4)$ & $82(2)$ \\
$\mathrm{C}(13)$ & $3525(6)$ & $12206(3)$ & $14380(3)$ & $55(1)$ \\
$\mathrm{C}(14)$ & $2670(10)$ & $10703(4)$ & $14366(4)$ & $86(2)$ \\
$\mathrm{C}(15)$ & $5614(9)$ & $12674(6)$ & $15004(4)$ & $95(2)$ \\
$\mathrm{C}(16)$ & $2180(10)$ & $12837(6)$ & $14959(4)$ & $96(3)$ \\
\hline
\end{tabular}

Equivalent isotropic $U$ defined as one third of the trace of the orthogonalized $U_{i j}$ tensor.

Kaburagi, Z.-Z. Sung and S.-W. Kim, Chem. Pharm. Bull., 39, 1132 (1991).

2. V. Prelog, O. Metzler and O. Jerger, Helv. Chim. Acta, 30,
Table 3 Geometric parameters

\begin{tabular}{|c|c|c|c|}
\hline \multicolumn{4}{|c|}{ Bond lengths ( $\AA$ ). } \\
\hline$O(1)-C(4)$ & $1.343 \quad(4)$ & $O(2)-C(7)$ & $1.193 \quad(5)$ \\
\hline$O(3)-C(7)$ & $1.308 \quad(5)$ & $\mathrm{O}(3)-\mathrm{C}(11)$ & $1.440 \quad(5)$ \\
\hline $\mathrm{O}(4)-\mathrm{C}(2)$ & $1.333 \quad(4)$ & $\mathrm{O}(5)-\mathrm{C}(8)$ & $1.224 \quad(4)$ \\
\hline$O(6)-C(8)$ & $1.317 \quad(4)$ & $\mathrm{O}(6)-\mathrm{C}(12)$ & $1.452 \quad(5)$ \\
\hline$O(7)-C(9)$ & $1.440 \quad(4)$ & $O(7)-C(10)$ & $1.328 \quad(4)$ \\
\hline $\mathrm{O}(8)-\mathrm{C}(10)$ & $1.184(4)$ & $C(1)-C(2)$ & $1.423 \quad(4)$ \\
\hline$C(1)-C(6)$ & $1.415 \quad(4)$ & $C(1)-C(8)$ & $1.467 \quad(5)$ \\
\hline$C(2)-C(3)$ & $1.403 \quad(5)$ & $C(3)-C(4)$ & $1.406 \quad(5)$ \\
\hline$C(3)-C(7)$ & 1.481 & $C(4)-C(5)$ & $1.392 \quad(4)$ \\
\hline$C(5)-C(6)$ & $1.368 \quad(4)$ & $C(6)-C(9)$ & $1.522(4)$ \\
\hline$C(10)-C(13)$ & $1.521 \quad(5)$ & $C(13)-C(14)$ & $1.530 \quad(5)$ \\
\hline$C(13)-C(15)$ & $1.504 \quad(7)$ & $\mathrm{C}(13)-\mathrm{C}(16)$ & $1.514(9)$ \\
\hline \multicolumn{4}{|c|}{ Bond Angles ( $\left(^{\circ}\right)$} \\
\hline$C(7)-O(3)-C(11)$ & $116.6(3)$ & $\mathrm{C}(8)-\mathrm{O}(6)-\mathrm{C}(12)$ & $116.7(3)$ \\
\hline$C(9)-O(7)-C(10)$ & $117.4(2)$ & $\mathrm{C}(2)-\mathrm{C}(1)-\mathrm{C}(6)$ & $118.5(3)$ \\
\hline$C(2)-C(1)-C(8)$ & $116.7(3)$ & $C(6)-C(1)-C(8)$ & $124.8(3)$ \\
\hline$O(4)-C(2)-C(1)$ & $121.2(3)$ & $\mathrm{O}(4)-\mathrm{C}(2)-\mathrm{C}(3)$ & 117.2 \\
\hline$C(1)-C(2)-C(3)$ & 121.5 & $C(2)-C(3)-C(4)$ & 117.7 \\
\hline $\mathrm{C}(2)-\mathrm{C}(3)-\mathrm{C}(7)$ & $125.0(3)$ & $C(4)-C(3)-C(7)$ & 117.4 \\
\hline $\mathrm{O}(1)-\mathrm{C}(4)-\mathrm{C}(3)$ & $123.8(3)$ & $\mathrm{O}(1)-\mathrm{C}(4)-\mathrm{C}(5)$ & $115.2(3)$ \\
\hline$C(3)-C(4)-C(5)$ & $121.0(3)$ & $C(4)-C(5)-C(6)$ & $121.6(3)$ \\
\hline$C(1)-C(6)-C(5)$ & $119.7(3)$ & $\mathrm{C}(1)-\mathrm{C}(6)-\mathrm{C}(9)$ & $122.0(3)$ \\
\hline$C(5)-C(6)-C(9)$ & 118.3 & $\mathrm{O}(2)-\mathrm{C}(7)-\mathrm{O}(3)$ & $120.6(3)$ \\
\hline $\mathrm{O}(2)-\mathrm{C}(7)-\mathrm{C}(3)$ & $122.4(3)$ & $\mathrm{O}(3)-\mathrm{C}(7)-\mathrm{C}(3)$ & $117.0(3)$ \\
\hline$O(5)-C(8)-O(6)$ & $120.7(3)$ & $\mathrm{O}(5)-\mathrm{C}(8)-\mathrm{C}(1)$ & $123.4(3)$ \\
\hline$O(6)-C(8)-C(1)$ & $115.9(3)$ & $\mathrm{O}(7)-\mathrm{C}(9)-\mathrm{C}(6)$ & $106.9(2)$ \\
\hline $\mathrm{O}(7)-\mathrm{C}(10)-\mathrm{O}(8)$ & $122.5(3)$ & $\mathrm{O}(7)-\mathrm{C}(10)-\mathrm{C}(13)$ & $112.9(3)$ \\
\hline$O(8)-C(10)-C(13)$ & $124.6(3)$ & $C(10)-C(13)-C(14)$ & 112.1 \\
\hline$C(10)-C(13)-C(15)$ & $107.4(3)$ & $\mathrm{C}(14)-\mathrm{C}(13)-\mathrm{C}(15)$ & $109.0(4)$ \\
\hline$C(10)-C(13)-C(16)$ & $108.3(4)$ & $\mathrm{C}(14)-\mathrm{C}(13)-\mathrm{C}(16)$ & $108.9(4)$ \\
\hline$C(15)-C(13)-C(16)$ & $111.2(4)$ & & \\
\hline
\end{tabular}

575 (1947); V. Prelog, L. Ruzicka and O. Metzler, Helv. Chim. Acta, 30, 1883 (1947).

3. H. Bertz, Synthesis, 1980, 708.

4. M. Harris, T. P. Murray, C. M. Harris and Gumulka, J. Chem. Soc. Chem. Commun., 1974, 362.

5. N. Takeuchi, K. Ochi, M. Murase and S. Tobinaga, $J$. Chem. Soc., Chem. Commun., 1980, 593.

6. W. Ried and W. Kunkel, J. Liebigs. Ann. Chem., 717, 54 (1968).

7. A. Covarrubias-Zúñiga, L. A. Maldonado and A. GonzálezLucas, submitted for publication.

8. G. M. Sheldrick (1990). SHELXTL/PC User's Manual. Siemens Analytical X-ray Instruments Inc., Madison Wisconsin, USA.

9. Siemens (1994). XSCANS User's Manual. Version 2.1. Siemens Analytical X-ray Instruments Inc., Madison, Wisconsin, USA.

(Received November 18, 1997) (Accepted February 16, 1998) 